\title{
Interpretação de um Teste sob a Visão Epidemiológica. Eficiência de um Teste
}

\author{
Takao Kawamura
}

Araçatuba, SP

\begin{abstract}
A evolução do raciocínio na interpretação dos fenômenos naturais, através dos tempos, trouxe, como conseqüência, as bases matemáticas do pensamento científico. $\mathrm{Na}$ medicina não foi diferente: a observação dos fenômenos biológicos, a procura de soluções para diminuir o impacto das doenças e a necessidade de se provar, cientificamente, a eficácia de métodos propedêuticos e de procedimentos terapêuticos abriu as portas para o que, hoje, se denomina medicina baseada em evidências.

Thomas Bayes, um matemático inglês do século XVII legou-nos o seu teorema que estabeleceu que a probabilidade pós-teste de uma doença era função da sensibilidade e especificidade do exame e da prevalência da doença na população (probabilidade pré-teste). Nós médicos, ao formularmos as nossas hipóteses diagnósticas, ao interpretarmos os exames laboratoriais e ao prescrevermos um tratamento, intuitivamente. utilizamos o teorema de Bayes. Hoje, vivemos a era da alta tecnologia em que as pessoas, freqüentemente, tendem a interpretar a positividade de um exame sofisticado e caro como sinônimo de doença. Não devemos esquecer que todos os exames, sem exceção, desde o corriqueiro exame clínico até uma tomografia computadorizada, estão limitados pela sensibilidade, especificidade e valor preditivo pré-teste.

Defenderemos, nesta apresentação, a introdução de um simples e novo conceito (baseado em antigos e conhecidos conceitos), que, provisoriamente, poderíamos denominá-lo de eficiência de um teste (Ef), como uma arma epidemiológica e propedêutica. Não encontramos nenhuma citação sobre o presente conceito proposto, tanto na literatura nacional como na de língua inglesa. Esta explicação tornarse-á mais didática se relembrarmos esses velhos conhecimentos básicos de epidemiologia clínica (tab. I).
\end{abstract}

Cardioclínica Araçatuba

Correspondência: Takao Kawamura - Rua Tiradentes, 1301 - 16015-020 Araçatuba, SP - E-mail: takaok@terra.com.br

Recebido para publicação em 10/5/01

Aceito em 5/9/01
Sensibilidade (s) - é a probabilidade de um indivíduo avaliado e doente de ter seu teste alterado (positivo).

$\mathbf{s}=$ número de indivíduos doentes e com teste positivo/número total de indivíduos doentes; ou:

$\mathbf{s}=\mathbf{V P} /(\mathbf{V P}+\mathbf{F N})$ (equação 1$)$

Especificidade (e) - é a probabilidade de um indivíduo avaliado e normal ter seu teste normal (negativo).

$\mathbf{e}=$ número de indivíduos normais e com teste negativo/número total de indivíduos normais; ou:

$\mathbf{e}=\mathbf{V N} /(\mathbf{V N}+\mathbf{F P})$ (equação 2)

Prevalência (p): é a fração de indivíduos doentes na população total avaliada.

$\mathbf{p}=$ número de indivíduos doentes / número de indivíduos da população; ou: $\mathbf{p}=\mathbf{D o} / \mathbf{n}$ (equação 3$)$ (onde: $\mathbf{D o}=$ doentes; $\mathbf{n}=$ população)

Valor preditivo positivo(VPP): é a probabilidade de um indivíduo avaliado e com resultado positivo ser realmente doente.

$\mathbf{V P P}=\mathbf{V P} /(\mathbf{V P}+\mathbf{F P})$ (equação 4$)$

Valor preditivo negativo (VPN): é a probabilidade de um indivíduo avaliado e com resultado negativo ser realmente normal.

$\mathbf{V P N}=\mathbf{V N} /(\mathbf{V N}+\mathbf{F N})($ equação 5$)$

A partir dos dados expostos podemos delinear as seguintes fórmulas:

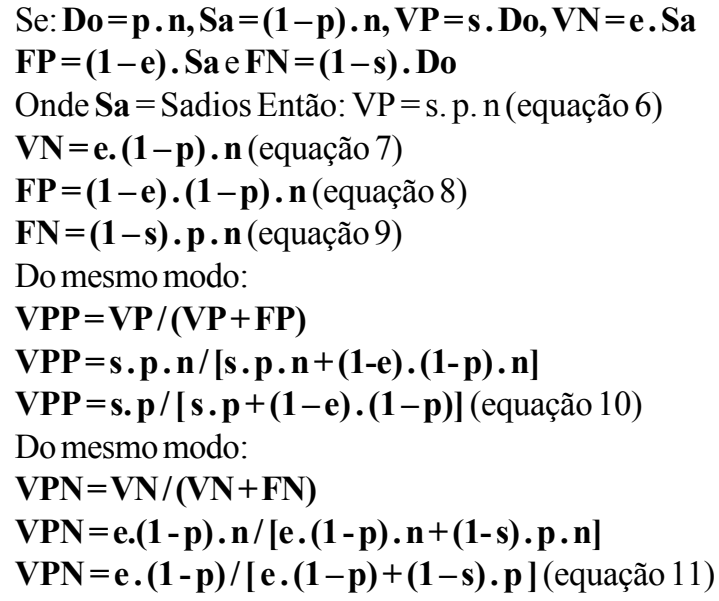




\begin{tabular}{|c|c|c|c|}
\hline \multirow[b]{2}{*}{ Teste } & \multirow[b]{2}{*}{$\begin{array}{l}\text { Positivo } \\
\text { Negativo }\end{array}$} & \multicolumn{2}{|c|}{ Doença } \\
\hline & & $\begin{array}{c}\text { Positivo } \\
\text { VP } \\
\text { FN }\end{array}$ & $\begin{array}{c}\text { Negativo } \\
\text { FP } \\
\text { VN }\end{array}$ \\
\hline $\begin{array}{l}\text { VP- } \\
\text { FN- f }\end{array}$ & $\begin{array}{l}\text { ositivo; V } \\
\text { fo. }\end{array}$ & o negativo & so positivo; \\
\hline
\end{tabular}

Esta "poluição de números" é indigesta para quem não gosta de matemática, mas é fundamental para o entendimento do raciocínio. O acompanhamento do leitor executando as operações matemáticas junto à leitura será extremamente elucidativo.

A indicação e a valorização de um exame para fins diagnósticos deverão ser regidas pela relação custo-benefício, levando-se em consideração o valor preditivo pré-teste (igual à prevalência da doença). Assim, a interpretação do seu resultado não pode estar divorciada de uma visão epidemiológica bem alicerçada. Recentemente, foi publicada na revista da SOCESP uma excelente revisão sobre o assunto ${ }^{1}$, da qual citaremos um estudo baseado em autópsias estratificando a prevalência da doença arterial coronariana ${ }^{2}$. Esses dados (tab. II) serão muito úteis para auxiliar nossos cálculos e aumentarão, significativamente, nosso poder diagnóstico.

Vamos analisar três situações comuns na prática clínica. Exemplo 1: mulher de 35 anos portadora de dor torácica não-anginosa é submetida a teste ergométrico para avaliar possibilidade de insuficiência coronariana.

Considerar a sensibilidade e a especificidade do teste ergométrico em $85 \%$ e $75 \%$, respectivamente. Consultando a tabela II, poderíamos assumir em $1 \%$ a prevalência da doença nesse subgrupo de pacientes. A partir desses dados poderíamos construir o seguinte gráfico: utilizando-se as equações 10 e 11 ou plotando-se os dados diretamente na figura 1, poderemos encontrar os seguintes valores: $\mathbf{V P P}=$ $3,3 \%$ e VPN $=99,8 \%$, significa que, se o teste ergométrico for positivo, existem $3,3 \%$ de chances da paciente realmente ser doente, contra 96,7\% (100-3,3\%) de ser normal, apesar do resultado ser positivo. Se o teste for negativo, existem $99,8 \%$ de chances da paciente ser normal contra $0,81 \%$ (100$99,8 \%$ ) de ser doente, apesar do resultado ser negativo. Em outras palavras: testando-se este tipo de paciente seriam necessários realizar 100 testes para diagnosticar insuficiência coronariana em apenas 3,3 pacientes, representando um

\begin{tabular}{|c|c|c|c|c|c|c|}
\hline \multicolumn{7}{|c|}{$\begin{array}{c}\text { Tabela II - Estratificação de prevalência da doença arterial coronariana. } \\
\text { Estimativa de probabilidade pré-teste }{ }^{2}\end{array}$} \\
\hline \multicolumn{3}{|c|}{$\begin{array}{c}\text { Dor torácica } \\
\text { não-anginosa }\end{array}$} & \multicolumn{2}{|c|}{ Angina atípica } & \multicolumn{2}{|c|}{ Angina típica } \\
\hline Idade & Homens & Mulheres & Homens & Mulheres & Homens & Mulheres \\
\hline $30-39$ & $5,2 \pm 0,8$ & $0,8 \pm 0,3$ & $21,8 \pm 2,4$ & $4,2 \pm 1,3$ & $69,7 \pm 3,2$ & $25,8 \pm 6,6$ \\
\hline $40-49$ & $14,1 \pm 1,3$ & $2,8 \pm 0,7$ & $46,1 \pm 1,8$ & $13,3 \pm 2,9$ & $87,3 \pm 1,0$ & $55,2 \pm 6,5$ \\
\hline $50-59$ & $21,5 \pm 1,7$ & $8,4 \pm 1,2$ & $58,9 \pm 1,5$ & $32,4 \pm 3,0$ & $92,0 \pm 0,6$ & $79,4 \pm 2,4$ \\
\hline $60-69$ & $28,1 \pm 1,9$ & $18,6 \pm 1,9$ & $67,1 \pm 1,3$ & $54,4 \pm 2,4$ & $94,3 \pm 0,4$ & $90,6 \pm 1,0$ \\
\hline
\end{tabular}

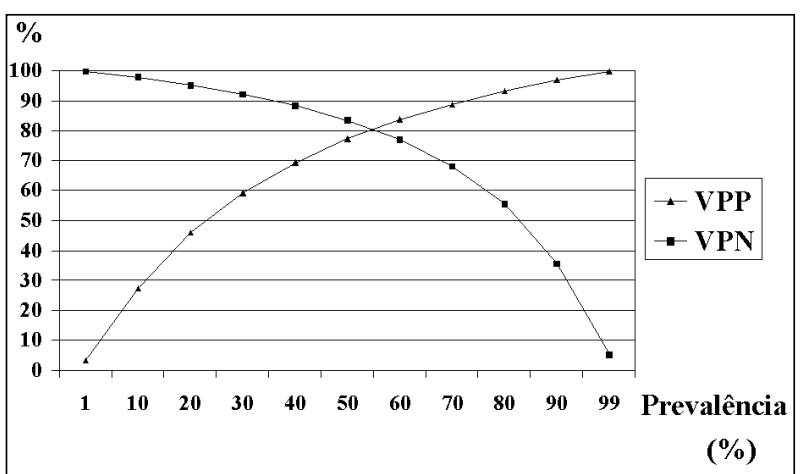

Fig. 1 - Valor preditivo positivo e valor preditivo negativo de um teste $(\mathrm{s}=85 \% \mathrm{e}=75 \%)$.

gasto muito grande de recursos e uma relação custo-benefício muito baixa.

Exemplo 2 - Homem de 65 anos de idade com dor precordial típica é submetido a teste ergométrico para avaliar possibilidade de insuficiência coronariana. Utilizando os mesmos artifícios do exemplo anterior teremos: $\mathbf{p}=\mathbf{9 4 \%}$, $\mathbf{V P P}=\mathbf{9 8} \%$ e $\mathrm{VPN}=\mathbf{2 4 \%}$. Isto significa que se o resultado do exame for positivo, há $98 \%$ de chances do indivíduo ser coronariano e $2 \%(100-98 \%$ ) de chances de ser normal. Se o teste for negativo, existem $24 \%$ de chances do indivíduo ser normal contra 76\% (100-24\%) de chances de ser coronariano, apesar do resultado ser negativo. Se a decisão de tratar ou de se indicar algum procedimento baseado apenas no resultado do exame, no caso de resultado negativo, existe uma grande chance de se tomar a decisão errada (de não tratá-lo/conduzi-lo como coronariopata).

Exemplo3-Homem de 55 anosdeidade com dorprecordial atípicaé submetido a teste ergométrico para avaliarpossibilidade de insuficiência coronariana. Utilizando-se os mesmos artifícios anteriores vamos encontrar os seguintes dados: $\mathbf{p}=\mathbf{6 0} \%, \mathbf{V P P}=$ $\mathbf{8 3 , 6 \%}$ e VPN $=\mathbf{7 6 , 9} \%$. Se o resultado do exame for positivo, há $83,6 \%$ de chances do indivíduo ser portador de doença contra 16,4\%(100-83,6\%); senegativo, há 76,9\%dechances do indivíduo sernormal contra23,1\%(100-76,9\%). Háuma discriminação bastante evidente, podendo a conduta tomada ser correta se baseada nos resultados do exame.

Dos três exemplos citados, poderíamos ainda extrair mais alguns dados interessantes: a tabela III demonstra claramente que o incremento do valor preditivo positivo pósteste foi maior no indivíduo de probabilidade pré-teste intermediária (exemplo 3). Nos dois extremos, o incremento foi muito pequeno (exemplos $1 \mathrm{e} 2$ ).

Existe, pois, uma zona média (de VPP pré-teste) onde o

\begin{tabular}{|c|c|c|c|}
\hline \multicolumn{4}{|c|}{$\begin{array}{l}\text { Tabela III - Análise do incremento no valor preditivo positivo } \\
\text { após realização do teste }\end{array}$} \\
\hline Exemplos: & VPP pré-teste (\%) & VPP pós-teste (\%) & $\mathrm{VPP}_{\text {pós }}-\mathrm{VPP}_{\mathrm{pré}}(\%)$ \\
\hline 1 & 1,0 & 3,3 & 2,3 \\
\hline 2 & 94,0 & 98,0 & 4,0 \\
\hline 3 & 60,0 & 83,6 & 23,6 \\
\hline
\end{tabular}


teste tem eficácia máxima e uma melhor relação custo-benefício. As questões seguintes a serem respondidas são: 1) que valores intermediários seriam esses? 2) Esses valores dependem da sensibilidade e especificidade do exame utilizado? 3) A partir de que valores, tanto para VPP como para VPN, poderíamos assumir como o de um exame eficiente? 4) E a que faixa de prevalência da doença (valor preditivo préteste) corresponderiam esses valores? 5) Essas faixas teriam a mesma extensão para qualquer exame? As respostas a essas questões pedem a definição de um novo conceito, como dito no início desta apresentação, baseado em velhos e conhecidos conceitos, que temporariamente o denominamos de eficiência de um teste (Ef).

Como vimos, todo exame tem a sua sensibilidade (s) e especificidade (e) próprias. Para cada conjunto de s e e poderíamos construir um gráfico relacionando VPP e VPN com p (prevalência). No exemplos anteriores de teste ergométrico onde $\mathbf{s}=85 \%$ e $\mathbf{e}=75 \%$ poderíamos construir um gráfico onde as curvas VPP e VPN teriam a mesma direção (para a direita), mas orientações diametralmente opostas (de baixo para cima e de cima para baixo, respectivamente). O cruzamento de ambas vai sempre ocorrer num ponto de prevalência média ( $60 \%$ no presente exemplo). Se tirarmos uma média de VPP e VPN obteremos valores que vão gerar uma terceira curva partindo de valores próximos a $50 \%$, subindo até a um pico pouco acima da média de $\mathbf{s}+\mathbf{e}$ (no nosso exemplo $85+75=80 \%$ ) que sempre corresponderá à interseção de VPP com VPN, e descendo depois para os valores iniciais (fig. 2). A esta altura, poderíamos denominar essa terceira curva de eficiência de um teste (Ef).

Assim: $\mathbf{E f}=(\mathbf{V P P}+\mathbf{V P N}) / \mathbf{2}($ equação 12$)$

Valores de prevalência em torno desse pico definiriam a zona de prevalência de máxima eficácia do exame (ZEf).

A questão a ser respondida agora é: como definir o que é eficiente e como calcular essas zonas de eficiência? Utilizando os mesmos artifícios utilizados na construção da figura 2, poderíamos fazer um gráfico de $\mathbf{s}=50 \% \mathrm{e} \mathrm{e}=50 \%$. O resultado seria a figura 3: visualmente podemos facilmente constatar que um exame com esta característica não acrescenta nada aos valores preditivos pré-teste, tendo portanto eficiência nula. Por outro lado, um exame hipotético (e pouco provável de existir algum dia) des $=100 \% \mathrm{e} e=100 \%$ geraria a figura 4 onde a eficiência seria a máxima. Poderíamos, por

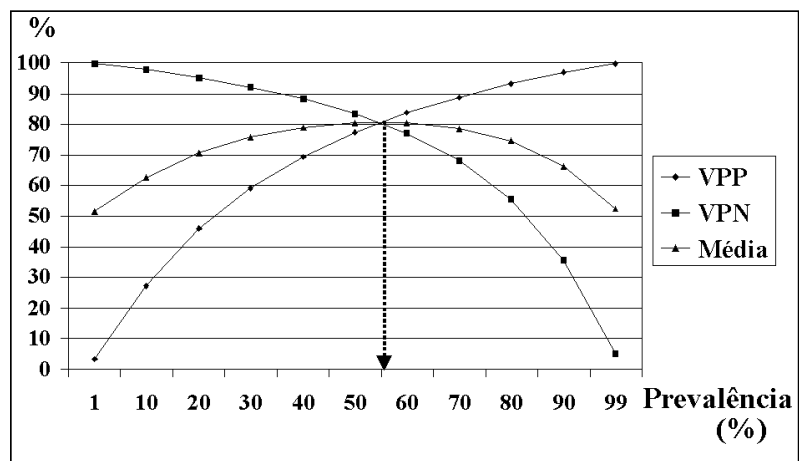

Fig. 2 - Cálculo da eficiência de um teste: média de VPPe VPN $(\mathrm{s}=85 \% \mathrm{e}=75)$.

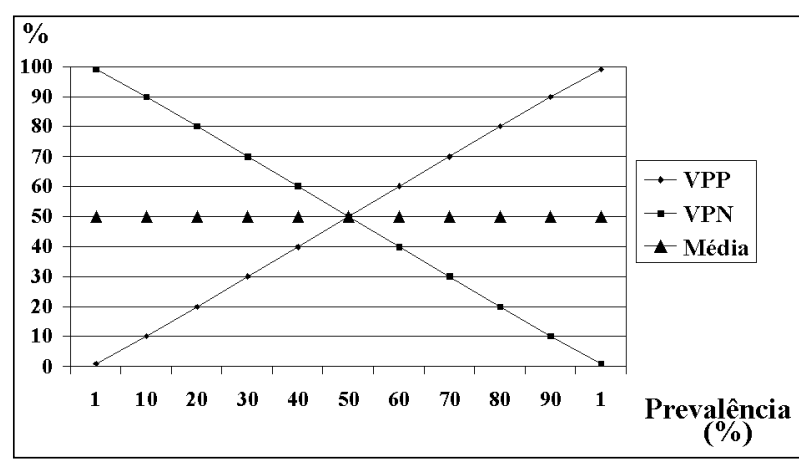

Fig. 3 - Cálculo de eficiência de um teste de $\mathrm{s}=50 \% \wedge \mathrm{e}=50 \%$.

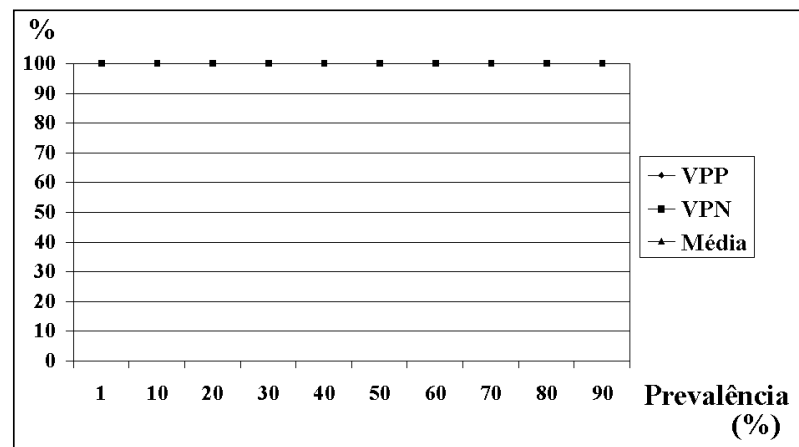

Fig. 4 - Cálculo de eficiência de um teste de $\mathrm{s}=100 \% \mathrm{e}=100 \%$. As três curvas (VPP VPN e Média) estão superpostas.

exemplo, assumir o valor $75 \%$ como valor de corte para estabelecer o que é eficiente, porém outros poderiam achar que $60 \%$ é aceitável e outros, mais prudentes, poderiam exigir $80 \%$. Seria aconselhável então subscrever essa sigla com um valor $\left(\mathbf{E f}_{\mathbf{7 5}}, \mathbf{E f}_{\mathbf{6 0}}, \mathbf{E f}_{\mathbf{8 0}}\right)$ de conveniência individual.

Vamos traduzir essas idéias na figura 5. Utilizando-se as fórmulas 10, 11 e 12 é possível construir-se as curvas do gráfico: curva de VPP(valor preditivo positivo) ascendente com concavidade para cima, VPN (valor preditivo negativo) descendente e com concavidade para cima e curva $\mathbf{E f}$ (eficiência de um teste) que é a média das outras duas anteriores: parte do valor pouco acima de $50 \%$, sobe progressivamente até um pico pouco acima da média de sensibilidade e especificidade ([s $+\mathbf{e}] / \mathbf{2}), 85 \%$ no nosso exemplo $([90+80] /$

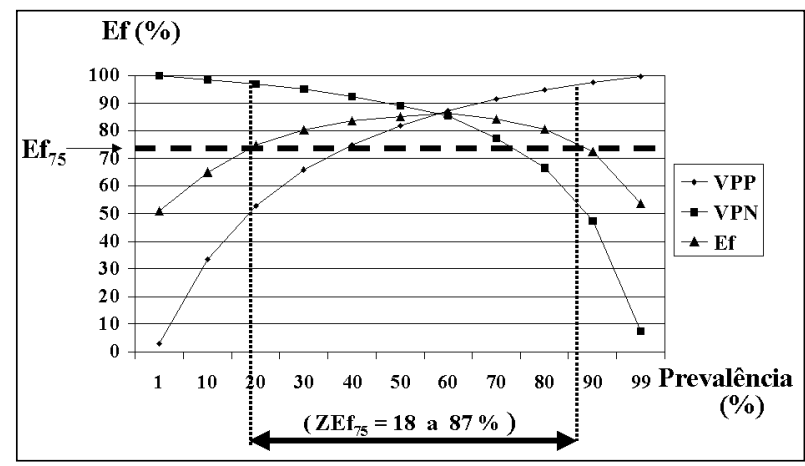

Fig. 5 - Cálculo da zona de prevalência de eficiência máxima $\left(\mathrm{ZEf}_{75}\right)$ de $\mathrm{Ef}_{75}$ de um teste coms $=90 \% \mathrm{e}=80 \%$. 
$2=85$ ) e desce para os valores próximos dos iniciais. Escolhendo-se $\mathbf{E} \mathbf{f}=\mathbf{7 5} \%$, traçaremos uma reta nesse nível, paralelamente ao eixo de prevalência (p). Nos pontos de interseção com curva de eficiência e projetando-se esses pontos no eixo de prevalência (p), estaremos delimitando a zona de prevalência de máxima eficiência $\left(\mathbf{Z E f}_{75}\right)$ para esse exame $\left(\mathbf{Z E f}_{\mathbf{7 5}}=\mathbf{1 1}\right.$ a 87\%) tendo como referência o nível escolhido para eficiência de $75 \%\left(\mathbf{E f}_{75}\right)$. Por esses dados podemos inferir que se trata de um exame bastante acurado (níveis altos de sensibilidade e especificidade) e eficiente, possuindo um espectro de eficiência bastante largo, ou seja, cobre populações de baixa/média até média/alta prevalência (11 a 87\%).

Dispondo de simples informações (sensibilidade, especificidade e prevalência) podemos avaliar o grau de confiabilidade e eficiência do exame e dispor de dados para comparar o alcance de diferentes exames. Para se ter uma melhor idéia comparativa do poder de cada exame, vamos criar a figura 6 com várias curvas de Ef(exames com sensibilidade e especificidade variáveis) e usar os mesmos artifícios utilizados no gráfico anterior:

As curvas A, B, C, D, E e F correspondem respectivamente a Ef de testes de médias de sensibilidade e especificidade $([\mathbf{s}+\mathbf{e}] / 2)$ de 50, 60, 70, 75, 80 e 90\%. Traçamos uma reta paralela ao eixo de prevalência (p) pois assumimos 75\% como ponto de corte para $\mathbf{E f}\left(\mathbf{E f}_{75}\right)$. Os pontos de interseção dessa reta com as várias curvas, projetados no eixo p, vão definir as zonas de máxima eficiência (ZEf) da população $\mathbf{p}$ para os exames correspondentes no ponto de corte escolhido $\left(\mathbf{Z E f}_{75}\right)$. Fica fácil concluir que exames A, B e C estão abaixo da reta $75 \%$ e são, portanto, pouco ou nada eficientes. $\mathrm{O}$ exame D seria eficiente apenas no pico onde ele toca a reta de corte limitando a sua ZEf a um ponto (50\%) de p. O exame E teria uma faixa maior de eficiência $(\mathbf{Z E f}=\mathbf{2 6} \mathbf{a} \mathbf{7 4 \%})$ e o exameF, uma faixa maior ainda (ZEf $=11$ a $89 \%)$.

Em termos clínicos e epidemiológicos, qual a importância desses conceitos? Ao estipularmos um nível de eficiência, podemos excluir de nossa prática clínica exames e procedimentos pouco eficientes. Por outro lado, poderemos alicerçar nossas condutas e decisões, interpretando corretamente o significado de um resultado de exame. Quanto maior a eficiência de um teste, maior será a população (zona de prevalência) que se beneficiará do mesmo. Decisões ba-

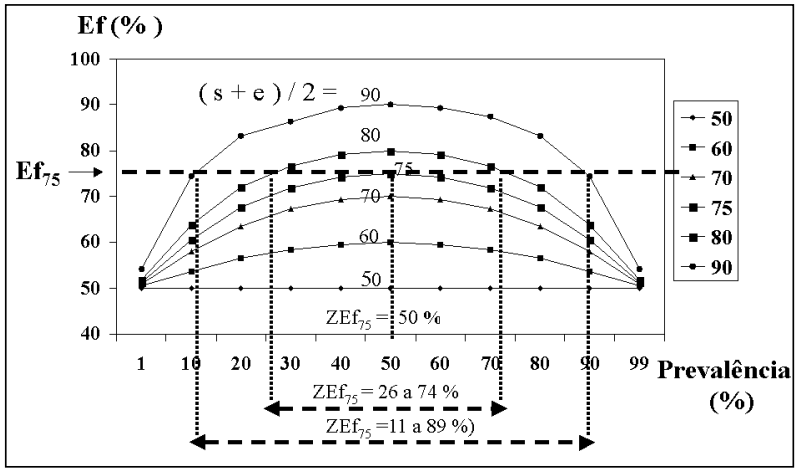

Fig. 6 - Comparação de curvas de eficiência de um teste (Ef) em situações variáveis de s e de e (de 50 a 90\%). Determinação de zonas de máxima eficiência (Zef). seadas em um raciocínio bem elaborado e ajustado em números confiáveis, seguramente, resultarão num grau maior de acerto, otimizando a relação custo-benefício. Acreditamos que todos os exames deveriam ter, nas suas conclusões, estipuladas a sua eficiência, a sua zona de melhor eficiência e a chance do paciente avaliado ter ou não doença de acordo com o resultado obtido. Se não for possível determinar a prevalência (valor preditivo pré-teste), então um gráfico com curvas de VPP, VPN e Ef deverá ilustrar o exame para que hipoteticamente o médico assistente possa calcular, de acordo com os dados clínicos disponíveis (por exemplo, no caso de coronariopatia: idade, sexo e característica da dor torácica), as chances de doença e normalidade.

Para ocálculodeprevalência(p)realizadonatabelaanterior, as fórmulas utilizadas foram: $\mathbf{A c}=(\mathbf{V P}+\mathbf{V N}) / \mathbf{n}$ (equação 13) onde: $\mathbf{A c}=$ acurácia e $\mathbf{n}=$ número total de exames ou população.

Utilizando-se as equações 6 e 7 e desenvolvendo a equação 13 teremos: $\mathbf{A c}=\mathbf{e}+\mathbf{p} \cdot(\mathbf{s}-\mathbf{e})($ equação 14) ou: $\mathbf{p}=$ $(\mathbf{A c}-\mathbf{e}) /(\mathbf{s}-\mathbf{e})$ (equação 15).

Se a sensibilidade (s) for igual à especificidade (e), então, para qualquer ponto de $\mathrm{p}$ (prevalência), a acurácia (Ac) será constante e igual ao valor de e.

Igualmente, se $\mathbf{s}<\mathbf{e}$, então Ac será máxima para os menores valores possíveis de $\mathrm{p}$ e cairá segundo uma reta descendente até atingir os menores valores para os máximos valores de $\mathrm{p}$.

Por outro lado, se $\mathbf{s}>\mathbf{e}$, então Ac será mínima para os menores valores possíveis de p e subirá segundo uma reta ascendente até atingir os maiores valores para os máximos valores de $\mathrm{p}$ (fig. 7).

Sabendo-se os valores de s, e e Ac é possível calcular a prevalência p de um determinado estudo (tab. IV).

Sob essa nova óptica também os livros de medicina estão sendo reescritos: conceitos, prevalências e tratamentos de doenças exigem hoje o rigor das evidências. É esta a impressão transmitida pela leitura dos primeiros capítulos de um tradicional livro de cardiologia em sua última edição ${ }^{3}$. Da mesma forma, números atribuídos para sensibilidade e especificidade dos habituais exames laboratoriais inexoravelmente terão de ser recalculados. Como isso tem sido feito até hoje? O Consenso da American College of Cardiology e American Heart Association para tomografia computadorizada por emissão de elétrons (EBCT: electron-beam computed tomography) selecionou 16 trabalhos de onde

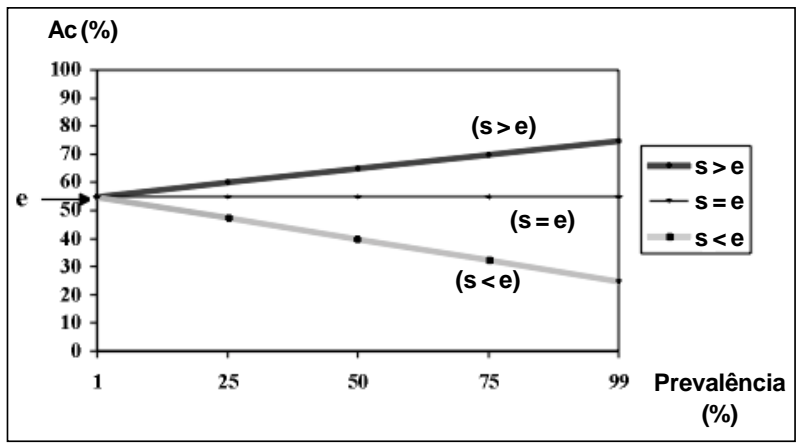

Fig. 7 - Acurácia(Ac), sensibilidade(s) e especificidade(e) de um teste e prevalência (p). 


\begin{tabular}{|c|c|c|c|c|}
\hline \multicolumn{5}{|c|}{$\begin{array}{l}\text { Tabela IV - Sensibilidade, especificidade e acurácia dos } 16 \\
\text { trabalhos selecionados no Consenso ACC/AHA para CT de } \\
\text { emissão de elétrons (EBCT) }{ }^{4} \text {. Cálculo de prevalência }\end{array}$} \\
\hline Estudo $\mathrm{Se}$ & $\begin{array}{l}\text { Sensibilidade } \\
(\%)\end{array}$ & $\begin{array}{c}\text { Especificidade } \\
(\%)\end{array}$ & $\begin{array}{l}\text { Acurácia } \\
(\%)\end{array}$ & $\begin{array}{c}\text { Prevalência } \\
(\%)\end{array}$ \\
\hline A & 88,4 & 100 & 90,7 & 80,2 \\
\hline B & 100 & 28,0 & 40,9 & 17,9 \\
\hline $\mathrm{C}$ & 100 & 47,2 & 72,0 & 47,0 \\
\hline D & 82,5 & 85,0 & 83,8 & 48,0 \\
\hline E & 92,8 & 66,7 & 82,5 & 60,5 \\
\hline $\mathrm{F}$ & 96,7 & 41,3 & 65,0 & 42,8 \\
\hline G & 91,0 & 90,0 & 90,4 & 40,0 \\
\hline $\mathrm{H}$ & 99,4 & 25.7 & 81,7 & 76,0 \\
\hline I & 92,5 & 72,7 & 88,2 & 78,3 \\
\hline $\mathrm{J}$ & 94,6 & 43,8 & 74,4 & 60,2 \\
\hline $\mathrm{K}$ & 70,0 & 71,0 & 70,9 & 10,0 \\
\hline $\mathrm{L}$ & 87,5 & 58,6 & 70,4 & 40,8 \\
\hline M & 94,7 & 21,4 & 51,1 & 40,5 \\
\hline $\mathrm{N}$ & 99,1 & 58,3 & 94,9 & 89,7 \\
\hline $\mathrm{O}$ & 95,6 & 30,5 & 58,4 & 42,9 \\
\hline $\mathrm{P}$ & 67,9 & 66,7 & 67,3 & 50,0 \\
\hline Total & 90,5 & 49,2 & 69,6 & 49,4 \\
\hline Média Pond. & d. 80,4 & 39,9 & 59,1 & 47,4 \\
\hline
\end{tabular}

extraiu valores médios de $90,5 \%$ e $49,2 \%$ para sensibilidade e especificidade, respectivamente ${ }^{4}$. Alguns desses dados estão ordenados na tabela IV que mostra uma grande variabilidade para sensibilidade (de 67,9 a 100\%) e de especificidade (de 21,4 a 90\%) dos trabalhos envolvidos. Quem está certo ou menos errado? Na realidade esses números traduzem diferentes níveis de corte (porcentagem de cálcio nas artérias coronárias) para "positivo" ou "negativo" e principalmente diferentes populações avaliadas (prevalências de 10 a $89,7 \%$ ). Isso leva também a resultados bastante variados e conseqüentemente a números errados.

Recentemente, um folheto direcionado a cardiologistas enaltecia as qualidades de um determinado procedimento diagnóstico para insuficiência coronariana, conferindolhe uma acurácia de pelo menos $90 \%$. Já vimos que acurácia não mede apenas a qualidade de um teste, mas depende também da população avaliada. Para este exame, admite-se valores aproximados de $90 \%$ e $50 \%$ para sensibilidade e especificidade, respectivamente. Utilizando-se as equações 14 ou 15 teremos:

$$
\begin{aligned}
& \mathbf{A c}=\mathbf{e}+\mathbf{p} .(\mathrm{s}-\mathrm{e}) \\
& \mathbf{9 0}=\mathbf{5 0}+\mathbf{p} .(\mathbf{9 0}-50) \text { assim: } p=100 \%
\end{aligned}
$$

Ou seja: apenas para uma população extremamente selecionada a afirmação é verdadeira. Se um paciente de $\mathbf{p}=$ $\mathbf{5 0 \%}$ (por exemplo: homem de 50 anos com dor precordial atípica) fosse submetido a esse exame o resultado seria diferente:

$$
\mathrm{Ac}=50+0,5 .(90-50)
$$

$\mathrm{Ac}=\mathbf{7 0 \%}$

O exame continua sendo bom e acurado, mas nós não podemos nos embriagar com a sedução dos números.

A exemplo dos atuais ensaios randômicos e multicêntricos para procedimentos terapêuticos, a medicina (medicina legal, inclusive) também exigirá números mais concisos e confiáveis para procedimentos diagnósticos e a epidemiologia clínica, com certeza, desenvolverá metodologia própria para recalcular esses números. A trilha a ser seguida deverá passar por um consenso de especialistas onde se determinarão a normatização de um nível de corte para "positivo" e "negativo", a escolha do padrão-ouro e a execução de um determinado número de exames (distribuição multicêntrica) em todas as camadas representativas de uma população.

Vivemos a década do consumidor, do aprimoramento das relações interpessoais e institucionais, da cobrança de resultados e de transparência no destino dos finitos recursos comuns e, muito provavelmente, exigências nesse sentido ocorrerão; creio que devemos nos antecipar e ir ao encontro dos anseios da nossa sociedade e desvendar as revelações do nosso tempo. Isso é justo, prático e muito prazeroso e contemplará quem praticar a boa medicina baseada em evidências.

\section{Referências}

1. Lagudis S. A probabilidade pré-teste e o resultado de exames complementares. Rev Soc Cardiol Estado de São Paulo 2001; 11: 15-20.

2. Diamond GA, Forrester JS. Analysis of probability as an aid in the clinical diagnosis of coronary artery disease. N Engl J Med 1979; 300: 1350-8.

3. Braunwald E, Zipes DP, Libby P. Heart Disease. A Textbook of Cardiovascular Medicine. $6^{\text {th }}$ edition. Philadelphia: WB Saunders Co., 2001.
4. O'Rourke RA, Brundage BH, Froelicher VF, et al. American College of Cardiology/American Heart Association Expert Consensus Document on Electron-Beam Computed Tomography for the Diagnosis and Prognosis of Coronary Artery Disease. J Am Coll Cardiol 2000; 36: 326-40. 